\title{
Capacitación docente, basada en la teoría Montessori, para atender la discapacidad intelectual leve en niños de Educación General Básica de Guayaquil 2021
}

\author{
Teacher training, based on Montessori theory, to attend to mild intellectual \\ disability in children of Basic General Education of Guayaquil 2021
}

DOI: $10.46932 / \operatorname{sfjdv3n1-015}$

Received in: Dec 30st, 2021

Accepted in: Jan 1th, 2022

\author{
Patricia Mercedes Tapia Macías \\ Aspirante a Doctora en Educación de la Universidad Cesar Vallejo, Piura-Perú \\ Escuela de Posgrado \\ Programa Académico de Doctorado en Educación
}

Universidad Cesar Vallejo Piura-Perú. Magíster en Ciencias Internacionales y Diplomacia. Abogada. General Elizalde 119 y Pichincha. Guayaquil, Ecuador.

E-mail: patty_tapia56@yahoo.com

\author{
César Balladares Atoche \\ Lcdo. en Educación \\ Doctor en Educación \\ Universidad Cesar Vallejo. Piura - Perú \\ calle Tupac Amaru 326 San Jacinto - Tumbes \\ E-mail: cballadaresa@ucvvirtual.edu.pe
}

\section{RESUMEN}

La presente investigación tuvo como propósito determinar que la capacitación docente basada en la teoría Montessori, influye en la atención de la discapacidad intelectual leve en niños de educación general básica de Guayaquil. Se sustentó teóricamente con los aportes de Montessori (1907), Lee (2018), Molina (2012, 2015), Chiavenato (2000), Rodríguez y Ramírez (1997), OMS (2021), AARM (2020),Tünnermann (2011), (Aubrey, 2010), UNESCO (2006), Cossentino (2005), Díaz (2002), Ministerio de Educación del Ecuador (2011), entre otros. La investigación se catalogó como experimental-aplicada, con nivel netamente explicativo. El diseño se clasificó como pre-experimental, con modelo pre-test/post-test con un solo grupo. La población estuvo constituida por 50 profesionales de educación general básica; debido al número finito y conocido de la población se empleó un censo poblacional. Como técnica de recolección de datos se utilizó la encuesta y como instrumento, el cuestionario. El análisis descriptivo de los datos se realizó mediante la confección de tablas de frecuencia absoluta (fa) y porcentaje (\%), medidas de posición (promedio, mediana, mínima y máxima), dispersión (desviación estándar) y varianza. Asimismo, se realizó un análisis inferencial acerca de la población, basándose en los datos recolectados y la contrastación de las hipótesis planteadas. Los resultados evidenciaron una diferencia significativa de 19,02 , entre los resultados del pre y post test, con un nivel de significancia de $0,000<0,05$. Por tanto, se aceptó la hipótesis de investigación (Hi) y se rechazó la hipótesis nula (Ho), llegando a la conclusión que la propuesta basada en teoría Montessori influye de forma significativa y positiva en la atención de la discapacidad intelectual leve en niños de educación general básica de Guayaquil. El presente estudio representa un gran aporte para el sistema educativo en lo concerniente al área de la discapacidad e inclusión. 
Palabras Clave: Discapacidad, Discapacidad Intelectual Leve, Teoría Montessori, educación general básica.

\begin{abstract}
The purpose of this research was to determine that teacher training based on Montessori theory influences the care of intellectual disability level in children of basic general education in Guayaquil. It was theoretically supported by the contributions of Montessori (1907), Lee (2018), Molina (2012, 2015), Chiavenato (2000), Rodríguez and Ramírez (1997), OMS (2021), AARM (2020), Tünnermann (2011), (Aubrey, 2010), UNESCO (2006), Cossentino (2005), Díaz (2002), Ministry of Education of Ecuador (2011), among others. The research was classified as experimental-applied, with a clearly explanatory level. The design was classified as pre-experimental, with a pre-test / post-test model with only one group. The population consisted of 50 basic general education professionals; Due to the finite and known number of the population, a population census was used. The survey was used as a data collection technique and the questionnaire as an instrument. The descriptive analysis of the data was carried out by making tables of absolute frequency (fa) and percentage (\%), position measurements (average, median, minimum and maximum), dispersion (standard deviation) and variance. Likewise, an inferential analysis was carried out about the population, property in the collected data and the contrasting of the hypotheses raised. The results showed a significant difference of 19.02, between the pre and post test results, with a significance level of $0.000<0.05$. Therefore, the research hypothesis (Hi) was accepted and the null hypothesis (Ho) was rejected, reaching the conclusion that the proposal based on Montessori theory has a significant and positive influence on the care of the intellectual disability level in children of basic general education of Guayaquil. The present study represents a great contribution to the educational system regarding the area of disability and inclusion.
\end{abstract}

Keywords: Disability, Mild Intellectual Disability, Montessori Theory, basic general education.

\title{
1 INTRODUCCIÓN
}

La Organización Mundial de la Salud (2020) señaló que, de cada siete individuos a nivel mundial, uno tiene algún tipo de discapacidad; esto representa el $15 \%$ del total de todas las personas. Hoy en día, la discapacidad está contemplada como un punto de amplio interés en lo que respecta a los derechos humanos. Según la Convención de las Naciones Unidas (art. 24): “los estados parte asegurarán el acceso de las personas con discapacidad, a la enseñanza primaria y secundaria inclusiva, gratuita y de calidad, en su lugar de origen y en igualdad de condiciones” (p. 19). En este orden, la UNESCO (2019) motivó la creación y puesta en práctica de normativas y estrategias que garanticen la igualdad de oportunidades, para todos, en materia educativa. Cabe destacar, que, entre los grandes problemas para la atención de las personas con discapacidad, está la capacitación docente del aula regular (Rivera y Núñez, 2016).

En este sentido, la Agencia Europea para el Desarrollo de la Educación Especial (2011), manifestó que el papel del maestro(a) y su formación para una educación más inclusiva es realmente importante. No obstante, países como Dinamarca, Suecia, entre otros, expresaron que la diversidad e inclusión son temas más desarrollados dentro del currículo de educación especial, que en cursos para docentes regulares; lo cual concordó con Rivera y Núñez (2016) citados con anterioridad. Asimismo, en América Latina, la 
UNESCO señaló que la atención educativa para las personas con discapacidad es deficitaria, sobre todo, en términos de recursos, infraestructuras y educadores preparados para tal fin (Romero y Lauretti, 2006). Desde una perspectiva nacional, a partir del año 2011, el Ministerio de Educación ecuatoriano abordó temas relacionados con la capacitación y sensibilización docente hacia la atención de las necesidades educativas especiales. Actualmente, continua la necesidad imperante de capacitar a más y más educadores en ejercicio de su profesión (Bedor, 2018), conociendo que 455.829 personas en Ecuador tienen algún tipo de discapacidad según el registro del 2019 del Consejo Nacional de Discapacidades (CONADIS) y de esta cifra, 101.973 (22.37 \%) es de discapacidad intelectual (Impoex, 2019). En este orden de ideas, Montessori desarrolló un método científico innovador basándose en sus investigaciones llevadas a cabo a niños de un psiquiátrico. Hoy, dicha estrategia está inmersa en distintas instituciones escolares de 110 países alrededor del mundo (Steverlynck, 2010). Por tal razón, la capacitación docente basada en la Teoría Montessori resultó idónea para trabajar en pro de los niños con discapacidad intelectual, tomando en cuenta, que esta pedagogía otorga valía a la atención individual, la adaptación del ambiente, la motivación para desarrollar la independencia y la comunicación. Es por todo lo descrito, que se dio respuesta a la siguiente interrogante: ¿De qué manera la capacitación docente basada, en la teoría Montessori, mejora la atención a niños con discapacidad intelectual leve en educación general básica de Guayaquil?

La investigación tuvo justificación teórica, considerando la importancia de aumentar conocimientos en cuanto a capacitación docente y discapacidad. También, la aplicación de la teoría Montessori como un modelo innovador, diferente, menos tradicional, que permitió visualizar desde otro punto de vista a la discapacidad. Además, la justificación metodológica se basó en la creación de dos cuestionarios pertenecientes a cada una de las variables. En cuanto a la contribución práctica, la investigación se orientó en la elaboración de planes para la adquisición de aprendizajes significativos de corte inclusivos. Su aporte social consistió en buscar soluciones a las dificultades para la atención de las personas con discapacidad dentro del contexto educativo, familiar y comunitario. Por otra parte, el Objetivo General de la investigación consistió en: Determinar que la capacitación docente basada en la teoría Montessori, influye en la atención de la discapacidad intelectual leve en niños de educación general básica de Guayaquil. Se consideraron los objetivos específicos siguientes: 1) Establecer que la capacitación docente basada en la teoría Montessori, influye en la atención de la discapacidad intelectual leve, a nivel escolar; 2) Demostrar que la capacitación docente basada en la teoría Montessori, influye en la atención de la discapacidad intelectual leve, a nivel familiar; 3) Comprobar que la capacitación docente basada en la teoría Montessori, influye en la atención de la discapacidad intelectual leve, a nivel comunitario. Como Hipótesis General: Existe relación significativa entre la capacitación docente, basada en la teoría Montessori, y la atención de la DIL en niños de educación general básica de Guayaquil. 


\section{METODOLOGÍA}

El tipo de investigación fue experimental-aplicada, donde Hernández et. al. (2010), expresan que este tipo de estudio implica el uso de una variable independiente (causa) y otra dependiente (efecto). Es una investigación cuantitativa con un nivel netamente explicativo. En cuanto al diseño, se clasificó como pre-experimental, con modelo pre-test/post-test con un solo grupo. La población estuvo conformada por 50 docentes de educación general básica; debido al número finito y conocido de la población se prescindió de la técnica de muestreo y se empleó un censo poblacional (Sabino, 1995). Los criterios de inclusión para formar parte de la muestra son los siguientes: docentes (hombres/mujeres) de educación general básica que trabajen con niños(as) que tengan diagnóstico de discapacidad intelectual leve y pertenezcan a escuelas regulares de la ciudad de Guayaquil.

Seguidamente, para llevar a cabo la recolección de datos, se usó una encuesta en forma escrita, conocida como cuestionario (Tamayo y Tamayo, 2003) bajo la modalidad de Likert, conformada por veintiséis (26) preguntas. Para determinar la validez de contenido se realizó mediante el juicio de cinco expertos, que comprobaron el grado de relevancia de los ítems, calificándolos en base a su significancia, redacción, y facilidad de comprensión. Para establecer la validez de constructo, se obtuvo mediante el análisis factorial de componentes principales, utilizando la matriz de correlaciones de Pearson, en función de las varianzas-covarianzas posterior a la aplicación de la prueba piloto en la Unidad Educativa Patria Ecuatoriana. Finalmente, con respecto a la confiabilidad, se empleó el Alpha de Cronbach, donde se obtuvo un alto grado de confiabilidad con un resultado de 0,831. Los datos recolectados fueron introducidos en una plantilla electrónica (software Excel, 2016) y transferidos para el programa Statistical Package for Social Sciences- SPSS, versión para Windows, para el respectivo análisis descriptivo e inferencial. Por último, se sometió el estudio al programa de evaluación de trabajos de investigación (Turnitin), promoviendo la política antiplagio.

\section{RESULTADOS}

\subsection{ANÁLISIS DESCRIPTIVO}

\section{Objetivo General:}

Determinar que la capacitación docente, basada en la teoría Montessori, influye en la atención de la discapacidad intelectual leve en niños de educación general básica de Guayaquil. 
Tabla 2 Niveles de la Variable Atención de la discapacidad intelectual leve

\begin{tabular}{|c|c|c|c|c|}
\hline \multicolumn{5}{|c|}{ Variable: Atención de la discapacidad intelectual leve } \\
\hline \multirow[t]{2}{*}{ Tipo } & \multicolumn{2}{|c|}{ Pre-test } & \multicolumn{2}{|c|}{ Post-test } \\
\hline & Frecuencia & Porcentaje & Frecuencia & Porcentaje \\
\hline BUENA & 8 & 16 & 50 & 100 \\
\hline REGULAR & 42 & 64 & 0 & 0 \\
\hline DEFICIENTE & 0 & 0 & 0 & 0 \\
\hline TOTAL & 50 & 100 & 50 & 100 \\
\hline
\end{tabular}

Fuente: cuestionario aplicado.

Figura 1 Niveles de la Variable Atención de la discapacidad intelectual leve

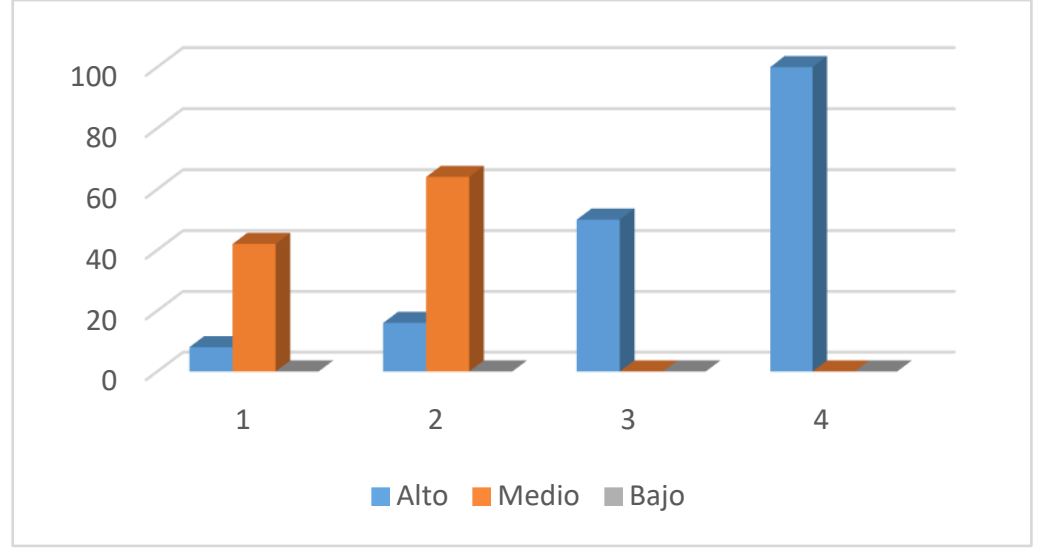

Interpretación: en la tabla 2 y figura 1 se observa que el $64 \%$ de los sujetos encuestados se posicionó en la clasificación con un tipo de atención regular, mientras que el 16\% se ubicó en el rango tipo de atención buena hacia las personas con discapacidad, durante el pre-test. Después de la puesta en marcha de la propuesta basada en la Teoría Montessori, la totalidad de la población tuvo un tipo de atención buena hacia las personas con discapacidad durante el postest.

\section{Objetivo Específico 1: Dimensión 1}

Establecer que la capacitación docente basada en la teoría Montessori, influye en la atención de la discapacidad intelectual leve, a nivel escolar.

Tabla 3 Niveles de la dimensión atención a nivel escolar

\begin{tabular}{|c|c|c|c|c|}
\hline \multicolumn{5}{|c|}{ Dimensión: Atención a nivel escolar } \\
\hline \multirow[t]{2}{*}{ Tipo } & \multicolumn{2}{|c|}{ Pre-test } & \multicolumn{2}{|c|}{ Post-test } \\
\hline & Frecuencia & Porcentaje & Frecuencia & Porcentaje \\
\hline BUENA & 12 & 24 & 50 & 100 \\
\hline REGULAR & 38 & 76 & 0 & 0 \\
\hline DEFICIENTE & 0 & 0 & 0 & 0 \\
\hline TOTAL & 50 & 100 & 50 & 100 \\
\hline
\end{tabular}

Fuente: cuestionario aplicado. 
Figura 2 Niveles de la dimensión atención a nivel escolar

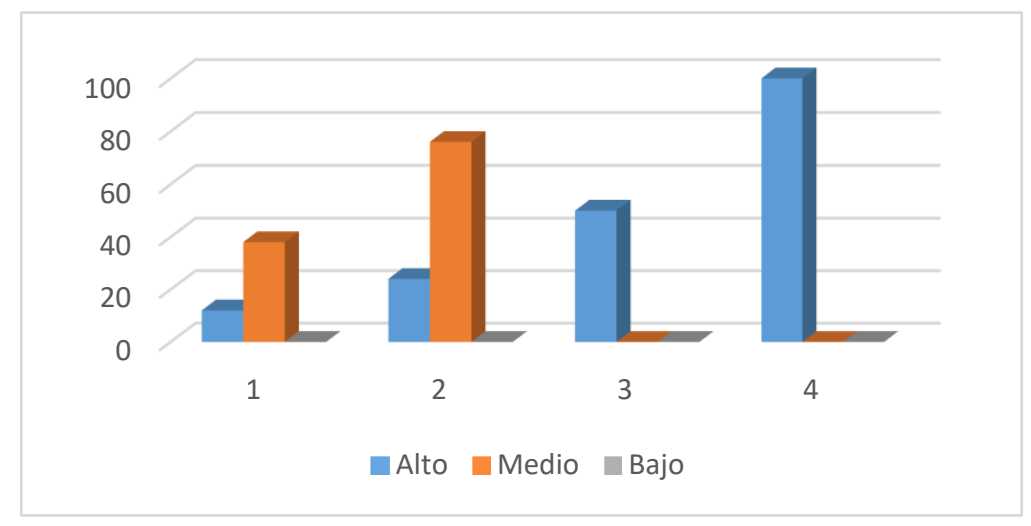

Interpretación: en la tabla 3 y figura 2 se aprecia que durante la aplicación del pretest el $76 \%$ de la población que participó en el estudio, se ubicó con un tipo de atención regular a nivel escolar; asimismo, el $24 \%$ estuvo en la categoría tipo de atención a nivel escolar buena hacia las personas con discapacidad. Luego de la aplicación de la propuesta basada en la Teoría Montessori, el 100\% se posicionó con un tipo de atención a nivel escolar buena durante la aplicación del postest.

Objetivo Específico 2: Dimensión 2

Demostrar que la capacitación docente basada en la teoría Montessori, influye en la atención de la discapacidad intelectual leve, a nivel familiar.

Tabla 4 Niveles de la dimensión atención a nivel familiar

\begin{tabular}{|c|c|c|c|c|}
\hline \multicolumn{5}{|c|}{ Dimensión: Atención a nivel familiar } \\
\hline \multirow[t]{2}{*}{ Tipo } & \multicolumn{2}{|c|}{ Pre-test } & \multicolumn{2}{|c|}{ Post-test } \\
\hline & Frecuencia & Porcentaje & Frecuencia & Porcentaje \\
\hline BUENA & 9 & 18 & 50 & 100 \\
\hline REGULAR & 38 & 76 & 0 & 0 \\
\hline DEFICIENTE & 3 & 6 & 0 & 0 \\
\hline TOTAL & 50 & 100 & 50 & 100 \\
\hline
\end{tabular}

Fuente: cuestionario aplicado.

Figura 3 Niveles de la dimensión atención a nivel familiar

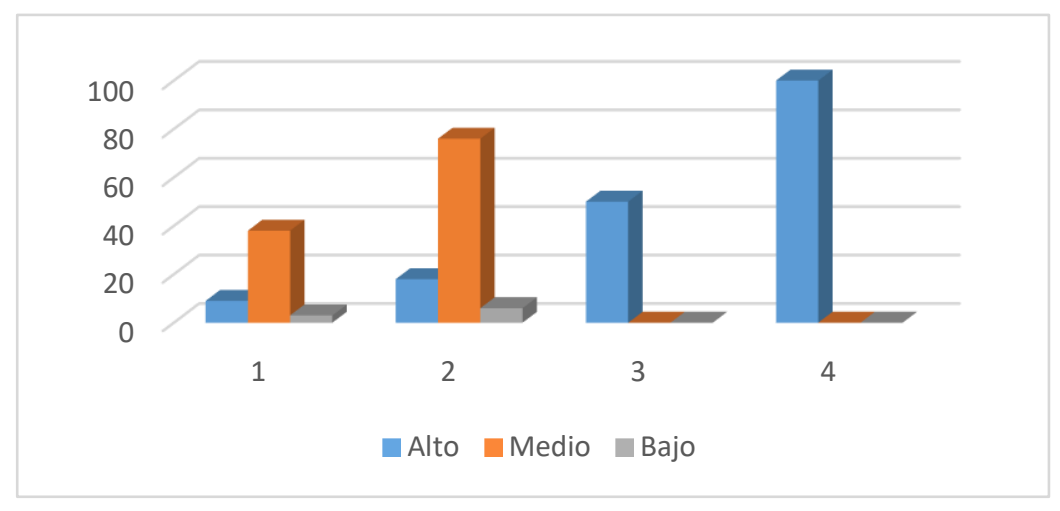


Interpretación: en la tabla 4 y figura 3 se evidencia durante la aplicación del pretest, que el 76\% de las personas participantes de la investigación, se encontraron en la clasificación con un tipo de atención regular a nivel familiar; de igual manera, el 18\% se ubicó en la tabla con un tipo de atención buena, mientras que el $6 \%$ se posicionó con un tipo de atención deficiente a nivel familiar hacia las personas con discapacidad. Posterior a la aplicación de la propuesta basada en la Teoría Montessori, todas las personas en el estudio (100\%) se ubicaron en un tipo de atención buena a nivel familiar durante la aplicación del postest.

\section{Objetivo Específico 3: Dimensión 3}

Comprobar que la capacitación docente basada en la teoría Montessori, influye en la atención de la discapacidad intelectual leve, a nivel comunitario.

Tabla 5 Niveles de la dimensión atención a nivel comunitario Dimensión: Atención a nivel comunitario

\begin{tabular}{|c|c|c|c|c|}
\hline \multicolumn{5}{|c|}{ Dimensión: Atención a nivel comunitario } \\
\hline \multirow{2}{*}{ Tipo } & \multicolumn{2}{|c|}{ Pre-test } & \multicolumn{2}{|c|}{ Post-test } \\
\hline & Frecuencia & Porcentaje & Frecuencia & Porcentaje \\
\hline BUENA & 5 & 10 & 50 & 100 \\
\hline REGULAR & 41 & 82 & 0 & 0 \\
\hline DEFICIENTE & 4 & 8 & 0 & 0 \\
\hline TOTAL & 50 & 100 & 50 & 100 \\
\hline
\end{tabular}

Fuente: cuestionario aplicado.

Figura 4 Niveles de la dimensión atención a nivel comunitario

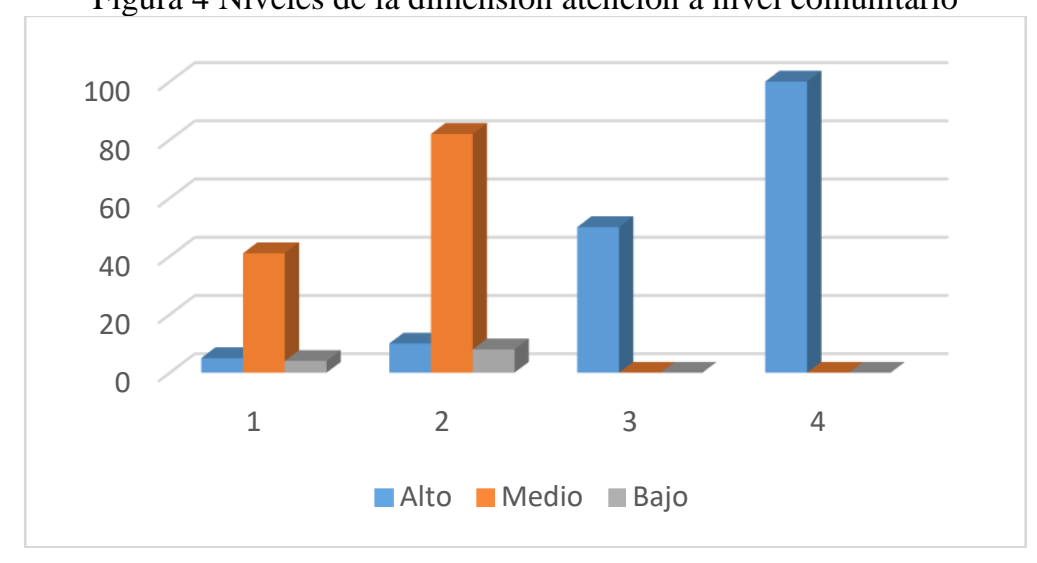

Interpretación: en la tabla 5 y figura 4 se muestra que durante la aplicación del pretest, el $82 \%$ de los educadores encuestados se ubicaron con un tipo de atención regular a nivel comunitario, el 10\% con un tipo de atención buena y el 8\% se ubicó en un tipo de atención deficiente hacia las personas con discapacidad. Ulterior a la aplicación de la propuesta basada en la Teoría Montessori, el 100\% se posicionó con un tipo de atención buena a nivel comunitario hacia las personas con discapacidad durante la aplicación del post-test. 
Tabla 6 Prueba de normalidad

Pruebas de normalidad

\begin{tabular}{|l|r|r|r|r|r|r|}
\hline & \multicolumn{3}{|c|}{ Kolmogorov-Smirnov $^{\mathrm{a}}$} & \multicolumn{3}{c|}{ Shapiro-Wilk } \\
\cline { 2 - 7 } & Estadístico & \multicolumn{1}{c|}{ gl } & \multicolumn{1}{c|}{ Sig. } & Estadístico & \multicolumn{1}{c|}{ gl } & Sig. \\
\hline $\begin{array}{l}\text { Pre test Atención a la } \\
\text { discapacidad intelectual- }\end{array}$ &, 172 & 50 &, 001 &, 869 & 50 &, 000 \\
$\begin{array}{l}\text { Postest Atención de la } \\
\text { discapacidad intelectual }\end{array}$ &, 157 & 50 &, 004 &, 967 & 50 &, 183 \\
\hline
\end{tabular}

a. Corrección de significación de Lilliefors

Debido a que la muestra es de 50 personas, se trabajó con Shapiro-Wilk.

\subsection{ANÁLISIS INFERENCIAL}

\section{Hipótesis General:}

Existe relación significativa entre la capacitación docente, basada en la teoría Montessori, y la atención de la discapacidad intelectual leve en niños de educación general básica de Guayaquil

Tabla 7 Estadística de la variable atención a la discapacidad intelectual Estadísticas de muestra única

\begin{tabular}{|l|r|r|r|r|}
\hline & $\mathrm{N}$ & Media & \multicolumn{1}{|c|}{$\begin{array}{c}\text { Desviación } \\
\text { estándar }\end{array}$} & \multicolumn{1}{|c|}{$\begin{array}{c}\text { Media de error } \\
\text { estándar }\end{array}$} \\
\hline $\begin{array}{l}\text { Atención a la discapacidad } \\
\text { intelectual Postest }\end{array}$ & 50 & 70,36 & 6,821 &, 665 \\
$\begin{array}{l}\text { Atención de la discapacidad } \\
\text { intelectual Pretest }\end{array}$ & 50 & 51,34 & 4,516 &, 639 \\
\hline
\end{tabular}

Tabla 8 Prueba de hipótesis de la variable atención a la discapacidad intelectual Estadísticos de prueba ${ }^{a}$

\begin{tabular}{|l|r|}
\hline & $\begin{array}{r}\text { Atención de la discapacidad } \\
\text { intelectual-Antes - Atención a la } \\
\text { discapacidad intelectual - Despues }\end{array}$ \\
\hline Z & $-6,146^{\mathrm{b}}$ \\
Sig. asintótica (bilateral) &, 000 \\
\hline
\end{tabular}

a. Prueba de rangos con signo de Wilcoxon

b. Se basa en rangos positivos.

Interpretación: como se observa en las tablas 7 y 8, existe diferencia significativa de 19,02, entre los resultados del pre y postest, el nivel de significancia es $0,000<0,05$, por tal razón, se acepta la hipótesis de investigación (Hi) y se rechaza la hipótesis nula (Ho), concluyéndose que la capacitación docente basada en la teoría Montessori generó efectos positivos en la atención de la discapacidad intelectual, en tanto los puntajes del postest fueron superiores al pretest. 
Hipótesis específica 1: Existe relación significativa entre la capacitación docente basada en la teoría Montessori, y la atención de la discapacidad intelectual leve, a nivel escolar.

Tabla 9 Estadística de la dimensión atención a nivel escolar Estadísticas de muestra única

\begin{tabular}{|l|r|r|r|r|}
\hline & $\mathrm{N}$ & Media & \multicolumn{1}{|c|}{$\begin{array}{c}\text { Desviación } \\
\text { estándar }\end{array}$} & \multicolumn{1}{|c|}{$\begin{array}{c}\text { Media de error } \\
\text { estándar }\end{array}$} \\
\hline $\begin{array}{l}\text { Atención a nivel escolar } \\
\begin{array}{l}\text { Postest } \\
\text { Atención a nivel escolar } \\
\text { Pretest }\end{array}\end{array} \quad 50$ & 37,90 & 3,489 &, 493 \\
\hline
\end{tabular}

Tabla 10 Prueba de hipótesis de la dimensión atención a nivel escolar Estadísticos de prueba ${ }^{a}$

\begin{tabular}{|l|r|}
\hline & $\begin{array}{r}\text { Atención a nivel escolar Pre test } \\
\text { Atención a nivel escolar postest }\end{array}$ \\
\hline $\mathrm{Z}$ & $-6,041^{\mathrm{b}}$ \\
Sig. asintótica (bilateral) &, 000 \\
\hline
\end{tabular}

a. Prueba de rangos con signo de Wilcoxon

b. Se basa en rangos positivos.

Interpretación: como se evidencia en las tablas 9 y 10, existe diferencia significativa de 9,65, entre los resultados del pre y postest, el nivel de significancia es $0,000<0,05$, por tal motivo, se acepta la hipótesis de investigación (Hi) y se rechaza la hipótesis nula (Ho), afirmándose que la capacitación docente basada en la teoría Montessori tuvo efectos positivos en la atención de la discapacidad intelectual a nivel escolar, puesto que los puntajes del postest fueron superiores al pretest.

Hipótesis específica 2: Existe relación significativa entre la capacitación docente basada en la teoría Montessori, y la atención de la discapacidad intelectual leve, a nivel familiar.

Tabla 11 Estadística de la dimensión atención a nivel familiar Estadísticas de muestra única

\begin{tabular}{|l|r|r|r|r|}
\hline & $\mathrm{N}$ & \multicolumn{1}{|c|}{ Media } & \multicolumn{1}{c|}{$\begin{array}{c}\text { Desviación } \\
\text { estándar }\end{array}$} & $\begin{array}{c}\text { Media de error } \\
\text { estándar }\end{array}$ \\
\hline $\begin{array}{l}\text { Atención a nivel familiar } \\
\begin{array}{l}\text { Postest } \\
\text { Atención a nivel familiar } \\
\text { Pretest }\end{array}\end{array} \quad 50$ & 19,22 & 1,993 &, 282 \\
\hline
\end{tabular}

Tabla 12 Prueba de hipótesis de la dimensión atención a nivel familiar Estadísticos de prueba ${ }^{a}$

\begin{tabular}{|l|r|}
\hline & $\begin{array}{r}\text { Atención a nivel familiar Pre test } \\
\text { Atención a nivel familiar Postest }\end{array}$ \\
\hline $\mathrm{Z}$ & $-6,099^{\mathrm{b}}$ \\
Sig. asintótica (bilateral) &, 000 \\
\hline
\end{tabular}

a. Prueba de rangos con signo de Wilcoxon

b. Se basa en rangos positivos. 
Interpretación: como se distingue en las tablas 11 y 12, existe diferencia significativa de 5,64, entre los resultados del pre y postest, el nivel de significancia es $0,000<0,05$, por ello, se acepta la hipótesis de investigación (Hi) y se rechaza la hipótesis nula (Ho), llegando a la conclusión que la capacitación docente basada en la teoría Montessori generó efectos positivos en la atención de la discapacidad intelectual a nivel familiar, debido a que los puntajes del postest fueron superiores al pretest.

Hipótesis específica 3: Existe relación significativa entre la capacitación docente basada en la teoría Montessori, y la atención de la discapacidad intelectual leve, a nivel comunitario.

Tabla 13 Estadística de la dimensión atención a nivel comunitario

Estadísticas de muestras emparejadas

\begin{tabular}{|c|c|c|c|c|c|}
\hline & & Media & $\mathrm{N}$ & $\begin{array}{c}\text { Desviación } \\
\text { estándar }\end{array}$ & $\begin{array}{l}\text { Media de error } \\
\text { estándar }\end{array}$ \\
\hline \multirow[t]{2}{*}{ Par 1} & $\begin{array}{l}\text { Postest dimensión Atención } \\
\text { a nivel comuniatrio }\end{array}$ & 13,24 & 50 & 1,965 & ,278 \\
\hline & $\begin{array}{l}\text { Pretest dimensión atención a } \\
\text { nivel comunitario }\end{array}$ & 9,42 & 50 & 1,617 & ,229 \\
\hline
\end{tabular}

Tabla 14 Prueba de hipótesis de la dimensión atención a nivel comunitario

Estadísticos de prueba ${ }^{a}$

\begin{tabular}{|l|r|}
\hline & $\begin{array}{r}\text { Atención a nivel comunitario Pretest } \\
\text { Atención a nivel comunitario Postest }\end{array}$ \\
\hline $\mathrm{Z}$ & $-5,723^{\mathrm{b}}$ \\
Sig. asintótica (bilateral) &, 000 \\
\hline
\end{tabular}

a. Prueba de rangos con signo de Wilcoxon

b. Se basa en rangos positivos.

Interpretación: como se visualiza en las tablas 13 y 14, existe diferencia significativa de 3,82, entre los resultados del pre y postest, el nivel de significancia es $0,000<0,05$, por tanto, se acepta la hipótesis de investigación (Hi) y se rechaza la hipótesis nula (Ho), concluyéndose que la capacitación docente basada en la teoría Montessori tuvo efectos positivos en la atención de la discapacidad intelectual a nivel comunitario, en tanto los puntajes del postest fueron superiores al pretest.

\section{DISCUSIÓN}

El trabajo de investigación desarrollado permitió comprobar que la capacitación docente basada en la teoría Montessori mejora de forma significativa la atención de la discapacidad intelectual leve en niños de educación básica general de Guayaquil - 2021.

Objetivo general: Determinar que la capacitación docente basada en la teoría Montessori, influye en la atención de la discapacidad intelectual leve en niños de educación básica general de Guayaquil. 
En las puntuaciones de la variable atención a la discapacidad intelectual leve en el pretest, el grupo se ubicó de forma predominante en la categoría atención regular con el 64\%. Por su lado, los puntajes del postest en el mismo grupo mejoraron al ubicarse el 100\% de los educadores encuestados, en un nivel de atención bueno, es decir, un incremento de $84 \%$ para el más alto nivel; de esta manera, se perciben los efectos positivos de la capacitación (tabla 2 y figura 1). En base a la aplicación de la de la prueba t. se puede evidenciar que existe diferencia significativa de 19,02, entre los resultados del pre y postest, el nivel de significancia es $0,000<0,05$, por tal motivo, se acepta la hipótesis de investigación (Hi) y se rechaza la hipótesis nula (Ho), derivándose que la capacitación docente basada en la teoría Montessori tuvo efectos positivos en la atención de la discapacidad intelectual, en tanto los puntajes del postest fueron mayores a los del pretest.

Dichos resultados concuerdan con la investigación de León (2017), denominada "La influencia de las actitudes de los profesores en el proceso de enseñanza-aprendizaje de estudiantes con Necesidades Educativas Especiales“. En este estudio, se observó la importancia que tiene capacitar al educador para que pueda desarrollar actitudes de corte inclusivas. Como complemento, Rivera y Núñez (2016) en su trabajo de grado titulado "Capacitación Profesional del Docente: Realidades de la Educación Inclusiva”, determinaron la relación, en alto grado, que existe entre la capacitación docente y los procesos de inclusión educativa. De igual manera, Viloria (2016), manifestó la relevancia de llevar a cabo transformaciones en favor de una educación más inclusiva en su estudio "La integración escolar del alumnado con Necesidades Educativas Especiales en el Municipio Caroní de Venezuela”.

Objetivo específico 1: Establecer que la capacitación docente basada en la teoría Montessori, influye en la atención de la discapacidad intelectual leve, a nivel escolar.

Con respecto a la atención de la discapacidad intelectual leve a nivel escolar, los resultados permitieron verificar durante el pretest que el $76 \%$ del total de la población, se encontraba en un tipo de atención regular, mientras que el $24 \%$ en un tipo de atención bueno. Posteriormente, al aplicar el postest, el 100\% se ubicó en un buen tipo de atención a nivel escolar hacia las personas con discapacidad, lo que es reflejo de los efectos positivos de la capacitación docente basada en la teoría Montessori. Al aplicar la estadística inferencial se denota que existe diferencia significativa de 9,65, entre los resultados del pre y postest, el nivel de significancia es $0,000<0,05$, por tanto, se acepta la hipótesis de investigación (Hi) y se rechaza la hipótesis nula (Ho), concluyéndose que la capacitación docente basada en la teoría Montessori generó efectos positivos en la atención de la discapacidad intelectual a nivel escolar, debido a que los puntajes del postest fueron superiores al pretest. Estos resultados se relacionan con lo expuesto por Umaña, et. al, (2020) en su investigación “Uso de las TIC en un Salón Montessori”, quienes plantearon que el acondicionamiento de espacios y los ajustes curriculares en función de la particularidad de cada 
estudiante con discapacidad intelectual leve son fundamentales para lograr una atención de calidad en la escuela. Asimismo, como lo manifestó Molina (2012), según la pedagogía Williams la educación no debe ser visualizada como un sistema estandarizado, sino, que los contenidos deben ser agradables para los educandos, por esto, la escogencia de recursos es de gran valor. Lo antes descrito, se relaciona con lo establecido por Montessori.

Objetivo específico 2: Demostrar que la capacitación docente basada en la teoría Montessori, influye en la atención de la discapacidad intelectual leve, a nivel familiar.

Tomando en consideración los resultados mostrados en la tabla 4 es posible distinguir que durante el pretest el 76\% de los docentes estaba en un tipo de atención regular hacia las personas con discapacidad, mientras que el 18\% se posicionó en un buen tipo de atención. En cambio durante la aplicación del postest, el 100\% de los educadores se ubicó en un tipo de atención bueno a nivel familiar. Lo anterior, es indicativo de que la capacitación docente, basada en la teoría Montessori, tuvo efectos positivos en la atención de la discapacidad intelectual leve a nivel familiar. La estadística inferencial permite observar que existe diferencia significativa de 5,64, entre los resultados del pre y postest, el nivel de significancia es 0,000 < 0,05, por ello, se acepta la hipótesis de investigación (Hi) y se rechaza la hipótesis nula (Ho), llegando a la conclusión que la capacitación docente basada en la teoría Montessori generó efectos positivos en la atención de la discapacidad intelectual a nivel familiar; en tanto los puntajes del postest fueron superiores al pretest. En este orden, los resultados concuerdan con el estudio de Altamirano (2018), llamado "Programa de acompañamiento a padres de familia para mejorar la inserción social de estudiantes con Necesidades Educativas Especiales asociadas a Discapacidad Intelectual”, donde expone de forma plena que la familia es una influencia significativa en el aprendizaje e inserción social de los estudiantes con discapacidad intelectual leve. También, para el Ministerio de Educación Ecuatoriano (2011) la participación familiar en el proceso de enseñanza-aprendizaje del educando con discapacidad intelectual es de gran trascendencia y por ello, deben buscarse mecanismos que lo garanticen.

Objetivo 3: Comprobar que la capacitación docente basada en la teoría Montessori, influye en la atención de la discapacidad intelectual leve, a nivel comunitario.

Los resultados observados en la tabla 5 y figura 4, evidencian que durante el pretest el $82 \%$ de la población encuestada, se encontraba dentro de la clasificación con un tipo de atención regular a nivel comunitario, mientras que el $10 \%$ se encontraba en un tipo de atención bueno; en cambio durante el postest, el $100 \%$ de los educadores se ubicaron en un tipo de atención bueno, lo que demuestra que la capacitación docente, basada en la teoría Montessori, tuvo efectos positivos en la atención a la discapacidad intelectual leve a nivel comunitario. De acuerdo con la estadística inferencial se puede 
apreciar que existe diferencia significativa de 3,82, entre los resultados del pre y postest, el nivel de significancia es $0,000<0,05$, por tal razón, se acepta la hipótesis de investigación (Hi) y se rechaza la hipótesis nula (Ho), llegando a la conclusión que la capacitación docente basada en la teoría Montessori generó efectos positivos en la atención de la discapacidad intelectual a nivel comunitario, en tanto los puntajes del postest fueron superiores al pretest. De esta manera, los datos recopilados concuerdan con lo establecido por Molina (2015) quien detalló que para la pedagogía Williams las instituciones escolares son un medio psicológico formado por diferentes personas, las cuales se dedican a la elaboración de actividades de agrado y voluntarias. Por tanto, debe abrirse para la comunidad y los museos, conciertos, foros u otros eventos de este tipo, son considerados como aulas. Aunado a lo expuesto, Montessori insistió en la importancia de que el docente se convirtiera en un vínculo entre el niño y su entorno (Lee, 2018).

\section{CONCLUSIONES}

La capacitación docente basada en la teoría Montessori, tiene efectos significativos en la atención de la discapacidad intelectual leve, esto se demuestra con la aplicación de la prueba t. en la que se visualiza que existe diferencia significativa de 19,02, entre los resultados pre y postest, el nivel de significancia es $0,000<0,05$. Por tal motivo, se acepta la hipótesis de investigación (Hi) y se rechaza la hipótesis nula (Ho), concluyéndose que el estudio tuvo efectos positivos, en tanto los puntajes del postest fueron mayores al pretest. Asimismo, la capacitación docente basada en la teoría Montessori, genera efectos significativos en la atención de la discapacidad intelectual leve a nivel escolar, esto se constata con la aplicación de la prueba t. en la que se distingue que existe diferencia significativa de 9,65, entre los resultados del pre y postest, el nivel de significancia es $0,000<0,05$. Por esto, se acepta la hipótesis de investigación (Hi) y se rechaza la hipótesis nula (Ho), derivándose que la propuesta tuvo efectos positivos en la atención de la discapacidad intelectual leve a nivel escolar, en tanto los puntajes del postest fueron superiores al pretest.

La capacitación docente basada en la teoría Montessori, tiene resultados significativos en la atención de la discapacidad intelectual leve a nivel familiar, esto se evidencia con la aplicación de la prueba t. en la que se puede observar que existe diferencia significativa de 5,64, entre los resultados pre y postest, el nivel de significancia es $0,000<0,05$. Así, se acepta la hipótesis de investigación (Hi) y se rechaza la hipótesis nula (Ho), determinándose que existen efectos positivos, debido a que los puntajes del postest fueron más altos que los del pretest. De igual modo, la capacitación docente basada en la teoría Montessori, produce efectos significativos en la atención de la discapacidad intelectual leve a nivel comunitario, esto se denota con la aplicación de la prueba t. en la que se aprecia que hay una diferencia significativa de 3,82, entre los resultados del pre y postest, el nivel de significancia es $0,000<0,05$. Así, se acepta la hipótesis de investigación (Hi) y se rechaza la hipótesis nula (Ho), visualizándose que tuvo efectos positivos a nivel comunitario. 


\section{REFERENCIAS}

Agencia Europea para el Desarrollo de la Educación del Alumnado con Necesidades Educativas Especiales (2011). Formación del profesorado para la educación inclusiva en Europa - Retos y oportunidades. Odense, Dinamarca: Agencia Europea para el Desarrollo de la Educación del Alumnado con Necesidades Educativas Especiales. ISBN (Impreso): 978-87-7110-203-1 ISBN (Electrónico): 97887-7110-224-6. Recuperado de:

https://www.google.com/url?sa=t\&rct=j\&q=\&esrc=s\&source=web\&cd=\&ved=2ahUKEwjTj86olPryAh VhSDABHYupCuIQFnoECAUQAQ\&url=https\%3A\%2F\%2Fwww.european-

agency.org\%2Fsites\%2Fdefault $\% 2$ Ffiles $\% 2$ Fte 4 i-synthesis-report-

es.pdf\&usg=AOvVaw3PNvcj4JgS9ld-A5HzTuyt

Altamirano, K. (2018). Programa de acompañamiento a padres de familia para mejorar la inserción social de estudiantes con necesidades educativas especiales asociadas a discapacidad intelectual, Trujillo - 2017. Universidad César Vallejo [Tesis doctoral]. Recuperado de: https://repositorio.ucv.edu.pe/handle/20.500.12692/22642?show=full

Arias, F. (2005). El Proyecto de Investigación: Introducción a la Metodología Científica. Caracas, Venezuela: Editorial Episteme, c. a.

Asociación Americana sobre Retraso Mental (2002). Definición de Discapacidad Intelectual. Recuperado de http://inico.usal.es/publicaciones/pdf/AAMR_2002.pdf

Asociación Internacional Montessori (2018). El Método Montessori. Fundación Argentina María Montessori. Recuperado de: https://www.fundacionmontessori.org// metodo-montessori.htm

Asociación Montessori de México (2021). Característica de una Guía Montessori. [Mensaje de un Blog]. Recuperado de: http://asociacionmontessori.mx/index.php/maria-montessori/que-es-una-guia-montessori

Avellaneda, C. y Ospina, L. (2018). Una mirada a textos sobre la pedagogía Montessori, principales aportes y su aplicabilidad en niños de 0 a 3 años. Universidad Católica de Pereira-Colombia. [Tesis de Maestría]. Recuperado de: http://hdl.handle.net/10785/4942

Bedor, L. (2018). La formación continua de los docentes para la inclusión de los estudiantes con necesidades educativas especiales. Ecuador, Guayaquil: Revista multidisciplinaria de Investigación Científica Espirales [Tesis de Doctorado]. Recuperado de: http://www.revistaespirales.com/index.php/es/article/view/338/302

Cabero, J. y Llorente M. (2013). La aplicación del juicio de experto como técnica de evaluación de las tecnologías de la información (TIC). En Edu-web. Revista de Tecnología de Información y Comunicación $\begin{array}{lllll}\text { en } & \text { Educación, } & 7 & \text { (2) } & \text { pp.11-22. }\end{array}$ http://tecnologiaedu.us.es/tecnoedu/images/stories/jca107.pdf

Campbell, D. y Stanley, J. (1978). Diseños experimentales y cuasiexperimentales en la investigación social. Buenos Aires: Amorrourtu Editores.

Chávez, N. (1994). Introducción a la investigación educativa. Maracaibo, Venezuela: Universidad del Zulia. 
Chiavenato, I. (2000). «Subsistema de Desarrollo de Recursos Humanos: Entrenamiento,» de Administración de Recursos Humanos. Santafé de Bogotá: Mc Graw Hill, p. 557.

Código de ética en investigación de la Universidad César Vallejo (23 de mayo de 2017). Resolución del Consejo Universitario N0126-2017/UCV. Trujillo, Perú. Recuperado de:

https://www.ucv.edu.pe/datafiles/C\%C3\%93DIGO\%20DE\%20\%C3\%89TICA.pdf

Díaz Barriga, F. (2002). Aportaciones de las perspectivas constructivista y reflexiva en la formación docente. Perfiles educativos 24 (97-98): 6-25.

Escuela Montessori Montevideo (2021). El Ambiente Preparado Montessori. [Mensaje de un Blog]. Recuperado de: https://escuelamontessori.edu.uy/ambiente-preparado-montessori/

Henríquez, E. (2010). Inclusión o integración de las personas con discapacidad. Universidad Nacional Experimental Rómulo Gallegos. [Monografías]. Recuperado de: https://www.monografias.com/trabajos82/inclusion-integracion-personas discapacidad/inclusionintegracion-personas-discapacidad3.shtml

Hernández, R., Fernández, C. \& Baptista, P. (2010). Metodología de la Investigación. (Quinta Edición). México D.F, México: McGraw-Hill.

Hernández-Nieto, R. (2011). Instrumentos de recolección de datos. Validez y Confiabilidad. Normas y Formatos. Mérida, Venezuela: Consejo de Estudios de Postgrados, Universidad de los Andes.

Inclúyeme.com (2021). Diferentes tipos de Discapacidad Intelectual. [Mensaje de un Blog]. Recuperado de: https://www.incluyeme.com/tag/discapacidad-intelectual-leve/.

Impoex Logística \& Comercio Exterior (5 de febrero de 2019). ¿Cuáles son los porcentajes de discapacidad en Ecuador? [Mensaje en un blog]. Recuperado de: https://www.impoex.ec/blog/cualesson-los-porcentaje-de-discapacidad-en-ecuador.

Lee, S. (2018). Diseño de un programa de formación docente para la atención de las necesidades educativas especiales. Pontificia Universidad Católica del Ecuador. [Tesis de Maestría]. Recuperado de: https://www.google.com/search?q=M\%C3\%89TODO+MONTESSORI+y+capacitacion+docente+disca pacidad+postgrado+tesis\&client=firefox $-b-$

d\&sxsrf=ALeKk02aspoPk6muOd5a1cZb4dwiM73JyA\%3A1621735414346\&ei=9repYNyxFMTp_Qb $\mathrm{U}-$

YkI\&oq=M\%C3\%89TODO+MONTESSORI+y+capacitacion+docente+discapacidad+postgrado+tesis \&gs_lcp=Cgdnd3Mtd216EAM6BwgjEK4CECdQ4D5YuUZg6ExoAXAAeAKAAfgDiAHdEZIBCzAu MS4zLjEuMS4xmAEAoAEBqgEHZ3dzLXdpesABAQ\&sclient=gwswiz\&ved=0ahUKEwjchKqa297wAhXEdN8KHdR8AgEQ4dUDCBA\&uact=5\#

León López, J. (2017) La influencia de las actitudes de los profesores en el proceso de enseñanzaaprendizaje de los alumnos con necesidades educativas especiales en República Dominicana. Universidad del País Vasco. [Tesis Doctoral]. Recuperado de: https://www.google.com/search?client=firefox-b$\mathrm{d} \& \mathrm{q}=+++++\mathrm{Le} \% \mathrm{C} 3 \% \mathrm{~B} 3 \mathrm{n}+\mathrm{L} \% \mathrm{C} 3 \% \mathrm{~B} 3 \mathrm{pez} \% 2 \mathrm{C}+\mathrm{J} .+\% 282017 \% 29+\mathrm{La}+$ influencia+de+las+actitudes+de + los+profesores+en+el+proceso+de+ense $\% \mathrm{C} 3 \% \mathrm{~B} 1$ anzaaprendizaje+de+los+alumnos+con+necesidades+educativas+especiales+en+Rep\%C3\%BAblica+Domin icana.+Universidad+del+Pa\%C3\%ADs+Vasco.+\%5BTesis+Doctoral\# 
Martín Arribas, M. (2004). Diseño y validación de cuestionarios. En Matronas Profesión, 5 (17), pp.2329. Recuperado de:

http://enferpro.com/documentos/validacion_cuestionarios.pdf

Méndez, A. (2002). Metodología, Diseño y Desarrollo del Proceso de Investigación. Bogotá, Colombia.

Ministerio de Educación del Ecuador (2011). Estrategias pedagógicas para atender a las necesidades educativas especiales en la educación regular. Vicepresidencia de la República del Ecuador. Quito: Editorial Ecuador. Recuperado de: https://es.slideshare.net/matalote77/manual-de-estrategiaspedaggicas-para-atender-nee

Molina, E. (2012). La experiencia Freinet en Guayaquil: Un análisis del currículo actual como base para una pedagogía popular. Revista Ciencia UNEMI, Número 8, año 5. pp. 36-45.

Molina, E. (2015). El Giro: una nueva categoría de la educación popular para la liberación de la pedagogía. En-claves del Pensamiento, vol. IX, núm. 18, julio-diciembre, 2015, pp. 79-101. Instituto Tecnológico y de Estudios Superiores de Monterrey Campus Ciudad de México Distrito Federal, México. Recuperado de:

https://www.google.com/url?sa=t\&rct=j\&q=\&esrc=s\&source=web\&cd=\&ved=2ahUKEwiA76mM3rnz AhVLVTABHSxIBrwQFnoECAUQAQ\&url=https\%3A\%2F\%2Fwww.redalyc.org\%2Fpdf\%2F1411\%2 F141143973004.pdf\&usg=AOvVaw0g2VkEYC-xVatUrBaTLGTI

Organización Mundial de la Salud (2011). Informe mundial sobre discapacidad. Ginebra: Suiza, OMS.

Organización Mundial de la Salud (1 de diciembre de 2020). 10 datos sobre la discapacidad. [Mensaje en un blog]. Recuperado de: https://www.who.int/es/news-room/facts-in-pictures/detail/disabilities.

Organización Mundial de la Salud (2021). Temas de Salud: Discapacidades. [Mensaje en un blog]. Recuperado de: https://www.who.int/topics/disabilities/es/

QuestionPro (2021). ¿Qué es la escala de intervalo? [Mensaje en un blog]. Recuperado de: https://www.questionpro.com/blog/es/escala-de-intervalo/

Rivera, D. y Núñez, C. (2016). Capacitación Profesional del Docente: Realidades de la Educación Inclusiva. Cuaderno de Pedagogía Universitaria Vol. 13 / no.26 / julio-diciembre 2016 / República Dominicana / PUCMM / ISSN 1814-4152 (en línea) / ISSN 1814-4144 (impresa) / p. 15-25. Recuperado de: http://cuaderno.pucmm.edu.do/

Rodríguez, M. y Ramírez, P. (1997). Administración de la Capacitación. México: McGraw-Hill.

Rodríguez, R. (2019). ¿Cuál es la influencia del método Montessori en la actualidad? Revista digital: La Mente es Maravillosa. Recuperado por: https://lamenteesmaravillosa.com/la-influencia-del-metodomontessori-en-la-actualidad/.

Romero, R. \& Lauretti, P. (2006). Integración educativa de las personas con discapacidad en Latinoamérica. Educere. Vol.10-N.33, ISSN 1316-4910. Universidad del Zulia, Maracaibo. Recuperado de: $h t t p: / / v e . s c i e l o . o r g / s c i e l o . p h p ?$ script $=$ sci_arttext\&pid=S1316-49102006000200019

Sabino, C. (1995). El proceso de investigación. Bogotá: Panamericana.

Solís, M. y Godoy, G. (2010). Impacto y necesidades de capacitación de docentes de educación básica, 
en relación con el currículo nacional básico. Informe Ejecutivo. Recuperado de: https://www.google.com/search?q=capacitacion+docente\&client=firefox

Steverlynck, A. (2010). La actualidad del Método Montessori. Argentina: Fundación Argentina María Montessori. Recuperado de: https://www.fundacionmontessori.org/metodo-montessori.htm

Shuttleworth, M. (12 de enero de 2009). Validez de criterio [Mensaje en un blog]. Jul 07, 2021. Recuperado de Explorable.com: https://explorable.com/es/validez-de-criterio

Tamayo y Tamayo, M. (1997). El Proceso de la Investigación Científica. México: Editorial Limusa S.A.

Tamayo y Tamayo, M. (2003) El proceso de la investigación científica. (4ta edición). México: Editorial Limusa.

Tünnermann Bernheim, C. (2011). El constructivismo y el aprendizaje de los estudiantes. Universidades, (48), 21-32[fecha de Consulta 9 de junio de 2021]. ISSN: 0041-8935. Disponible en: https://www.redalyc.org/articulo.oa?id=37319199005

Umaña, M.; Miranda, C.; y González, F. (2020). Uso educativo de TIC en un salón Montessori: diálogo entre la tecnología digital y los ritos de interacción social en el aula. Universidad de Chile [Tesis de Doctorado]. Rev. estud. exp. educ. vol.19 no.41 Concepción dic. 2020. Recuperado de:

http://dx.doi.org/10.21703/rexe.20201941umana2

UNESCO (2006). Educación Inclusiva. [Mensaje de un Blog]. Recuperado de: http://www.inclusioneducativa.org/ise.php?id=1

UNESCO (2019). Educación para personas discapacitadas. [Mensaje en un blog]. Recuperado de: https://es.unesco.org/themes/inclusion-educacion/personas-discapacitadas.

Viloria, M. (2016). La integración escolar del alumnado con necesidades educativas especiales en el municipio Caroní de Venezuela. Universidad de Girona, España. [Tesis Doctoral]. Recuperado de: https://www.tdx.cat/handle/10803/394056. 